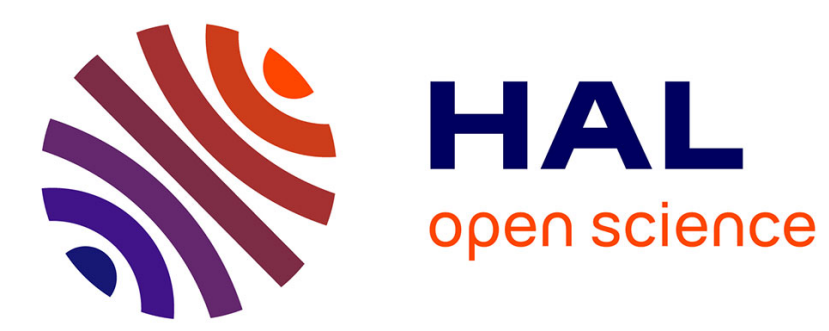

\title{
Optimal Balancing of Road Traffic Density Distributions for the Cell Transmission Model
}

Dominik Pisarski, Carlos Canudas de Wit

\section{To cite this version:}

Dominik Pisarski, Carlos Canudas de Wit. Optimal Balancing of Road Traffic Density Distributions for the Cell Transmission Model. CDC 2012 - 51st IEEE Conference on Decision and Control, Dec 2012, Maui, Hawaii, United States. hal-00727783

\section{HAL Id: hal-00727783 \\ https://hal.inria.fr/hal-00727783}

Submitted on 4 Sep 2012

HAL is a multi-disciplinary open access archive for the deposit and dissemination of scientific research documents, whether they are published or not. The documents may come from teaching and research institutions in France or abroad, or from public or private research centers.
L'archive ouverte pluridisciplinaire HAL, est destinée au dépôt et à la diffusion de documents scientifiques de niveau recherche, publiés ou non, émanant des établissements d'enseignement et de recherche français ou étrangers, des laboratoires publics ou privés. 


\title{
Optimal Balancing of Road Traffic Density Distributions for the Cell Transmission Model
}

\author{
Dominik Pisarski and Carlos Canudas-de-Wit
}

\begin{abstract}
In this paper, we study the problem of optimal balancing of traffic density distributions. The optimization is carried out over the sets of equilibrium points for the Cell Transmission Traffic Model. The goal is to find the optimal balanced density distribution that maximizes the Total Travel Distance. The optimization is executed in two steps. At the first step, we consider a nonlinear problem to find a uniform density distribution that maximizes the Total Travel Distance. The second step is to solve the constrained quadratic problem to find the near balanced optimal equilibrium point. At both steps, we use decomposition methods. The quadratic optimization problem is solved by using the Dual Problem. The computational algorithms associated to such a problem are given.
\end{abstract}

\section{INTRODUCTION}

Optimization of freeway traffic is nowadays one of the most intensively studied issues in transportation systems. A good number of both ramp metering and variable speed limiting methods have been developed and put into practice. The impact of these methods on economics, human leaving and nature is unquestionable. Shortened delays, reduced pollution, decreased number of accidents are among many other benefits, that should be mentioned here.

A variety of cost functions have been proposed in order to optimize the freeway traffic. The most common in use are the Total Travel Spent (TTS) and the Total Travel Distance (TTD), usually combined together [1]. The former is to decrease the time of travel incurred by all users of a freeway. This is achieved by reducing the vehicle density to the value that provides the free flow state. In turn TTD can be related to the usage of a freeway. The goal is to increase this usage, and this is obtained by flow maximization. TTS and TTD are often mixed with some extra terms penalizing abrupt variations in ramp metering and speed limiting signals [2].

Most of the optimization methods used in freeway traffic systems lead to open-loop solutions (see [3], [4]) that usually are implemented in the Model Predictive Control procedures [5]. In [6] authors presented an approach to approximate the optimal solution by using a neural network. The resulting system had a close-loop form. Another close-loop control method was proposed in [7], where authors used Powell's method to tune regulator parameters.

Dominik Pisarski is with NeCS team INRIA, Grenoble, France, dominik.pisarski@inria.fr

C. Canudas-de-Wit is a director of reearch at the CNRS, GIPSA-Lab. NeCS team, Grenoble, France, carlos.canudas-de-wit@gipsa-lab.grenobleinp.fr
Concerning the freeway traffic as a network system, we can distinguish two types of controllers i.e. centralized and distributed. To the centralized we include all controllers using the whole information collected from a freeway. Most of the gradient-based algorithms (including MPC) require this global information. To the distributed methods, we can include ALINEA [8], the demand-capacity and the occupancy strategies [9]. The aim for all of these methods is to keep the freeway condition closed to some pre-determined value. The controllers use real-time measurements. The other distributed method was presented in [10]. The authors applied sliding mode technique in order to prevent the propagation of shock waves. The controller uses only neighboring cellular information.

In this paper, we address the problem of balancing of density for the freeway traffic. By the balancing, we mean to find the best possible uniform distribution of vehicles along a freeway. Best stands here for the one, that optimizes some objective. The balancing should be considered as a promising objective for freeway control systems and this is due to the following facts. In practice, uniform density distribution on a freeway can be regarded as an equal inter-distance between vehicles. The driving experience allows as to claim that an equal inter-distance makes travel more smoothly, comfortable and safety. Smooth driving saves energy and reduces pollution. Comfort is one of the missing points in the standard objectives. In a fact, its lack impacts on the number of risky drivers' behaviours and accidents and therefore, it should be taken into consideration. We also believe that the balanced density can increase the capacity of a freeway. The concept of balancing has been already studied in other fields, for example in battery recharging processes [11], but for our best knowledge, it has not been introduced yet into the freeway traffic systems.

It was proven in [12] that a traffic freeway system described by the Cell Transmission Model is asymptotically stable and consequently, for every fixed input it converges to some equilibrium state. Therefore, we can design desired density equilibria by finding the corresponding input flows. Then the question arises. What is the input that guarantees the balanced equilibrium?

Authors of this paper presented preliminary study on density balancing in [13]. The goal was to analyse the structure of equilibrium sets and derive conditions for the existence of balanced equilibria. It was shown that for the free flow balanced equilibria some inequality relation between veloc- 


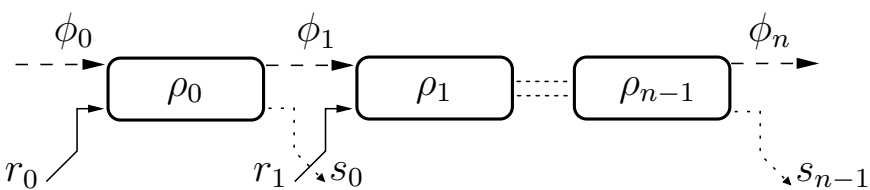

Fig. 1. Freeway divided into n sections. Each section is accompanied with at most one on-ramp and one off-ramp.

ities and split ratios must hold. For the special case, where all cells of a freeway are given with equal parameters and there is no off-ramps, the balanced equilibrium is possible only if the first on-ramp is serving cars to a freeway while all other are closed.

The contributions of this paper are twofold. The first is to determine the optimal balanced density in terms of TTD. Here, we will look for the optimal solution in the set of all possible states. The second is to find the equilibrium point that approaches this optimal uniform density distribution. For the optimization problem, we will use a convex formulation. The potential extension will be to solve the problem in a distributed manner.

In this paper, a method for solving the posed optimization problems is given. The ongoing research topic of the authors is to apply these algorithms for the model of Grenoble South Ring in the context of Grenoble Traffic Lab [14].

\section{The Cell Transmission Model With I/O}

In this paper, we consider a part of a freeway divided into sections (or cells) as depicted in Fig. 1. Each section is assumed to be equipped with at most one on-ramp and one off-ramp. For such a system, we adopt Daganzo's Cell Transmission Model (CTM), introduced in [15].

For the section $i$ we assume the following notation: $\rho_{i}-$ vehicle density, $\phi_{i}$-mainstream flow entering the section, $r_{i}-$ on-ramp flow entering the section, $s_{i}$-off-ramp flow leaving the section, $v_{i}$-free flow speed, $w_{i}$-congestion wave speed, $F_{i}$-capacity, $\bar{\rho}_{i}$-jam density, $l_{i}$-cell length. Nonlinear relation between flow and density is represented by the triangular fundamental diagram (see Fig. 2).

CTM describes the evolution of the system in discrete time $t_{k}=k \Delta t$, with $k \in \mathbb{N}$. Time step $\Delta t>0$ is taken small enough to provide convergence condition (for details see [16]). For the section $i$ the governing equation is:

$$
\rho_{i}(k+1)=\rho_{i}(k)+\frac{\Delta t}{l_{i}}\left[\Phi_{i}^{+}(k)-\Phi_{i}^{-}(k)\right] .
$$

Here $\Phi_{i}^{+}(k)$ and $\Phi_{i}^{-}(k)$ are total flows entering and leaving the section $i$, i.e.:

$$
\begin{aligned}
& \Phi_{i}^{+}(k)=\phi_{i}(k)+r_{i}(k), \\
& \Phi_{i}^{-}(k)=\phi_{i+1}(k)+s_{i}(k) .
\end{aligned}
$$

By introducing Demand $D_{i}$ and Supply $S_{i}$ functions:

$$
\begin{aligned}
& D_{i}(k)=\min \left\{\bar{\beta}_{i} v_{i} \rho_{i}(k), F_{i}\right\}, \\
& S_{i}(k)=\min \left\{w_{i}\left(\bar{\rho}_{i}-\rho_{i}(k)\right), F_{i}\right\}
\end{aligned}
$$

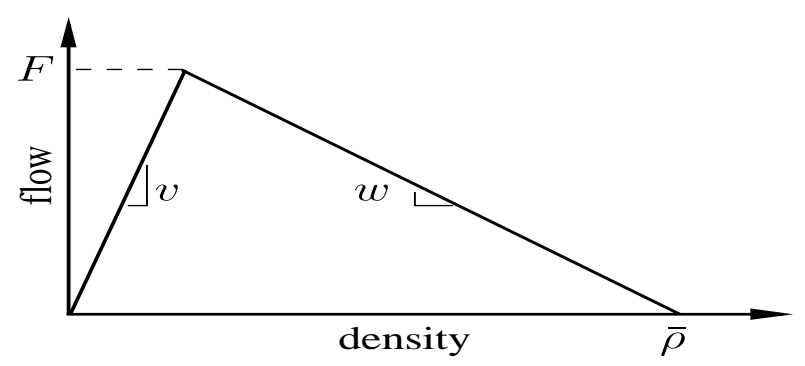

Fig. 2. The fundamental diagram. The shape is determined by the parameters: $F$ - capacity, $v$ - free flow velocity, $w$ - congestion wave speed.

according to Daganzo's merge connection model mainstream and on-ramp flows for $i=0,1, \ldots, n-1$ are given as follows:

$$
\begin{aligned}
& \text { if } D_{i-1}(k)+u_{i}(k) \leq S_{i}(k): \\
& \phi_{i}(k)=D_{i-1}(k), r_{i}(k)=u_{i}(k) \\
& \text { otherwise : } \\
& \phi_{i}(k)=\operatorname{mid}\left\{D_{i-1}(k), S_{i}(k)-u_{i}(k),\left(1-p_{i}\right) S_{i}(k)\right\}, \\
& r_{i}(k)=\operatorname{mid}\left\{u_{i}(k), S_{i}(k)-D_{i-1}(k), p_{i} S_{i}(k)\right\} .
\end{aligned}
$$

Here the function mid $\{\cdot\}$ returns the middle value. In (4) we introduced the on-ramp demands $u_{i}$ that will appear later as the inputs for the control system. By $p_{i} \in[0,1]$ we denote a parameter that captures the priorities between mainstream flow $\phi_{i}$ and on-ramp flow $r_{i}$ when merging in a section during the congestion. In order to determine the value for $p_{i}$ we should consider geometrical properties of on-ramp as well as drivers' behaviour. For the most of the cases, we can assume, that the priority is for the mainstream flow, and therefore we set $p_{i}<0.5$.

The boundary conditions are determined by some demand function $\bar{D}(k)=D_{-1}(k)$ for the section 0 and some supply function $\bar{S}(k)=S_{n}(k)$ for the section $n-1$. For the flow $\phi_{n}$ we adopt the simple connection model, and therefore:

$$
\phi_{n}(k)=\min \left\{D_{n-1}(k), \bar{S}(k)\right\} .
$$

Finally, for the off-ramp flows we assume:

$$
s_{i}(k)=\frac{1-\bar{\beta}_{i}}{\bar{\beta}_{i}} \phi_{i+1}(k) .
$$

Here $\bar{\beta}_{i}=1-\beta_{i}$, where $\beta_{i} \in[0,1)$ stands for the split ratio, which is the parameter that specifies a number of vehicles leaving the section $i$.

For the convenience of our further investigation, we rewrite the governing equation (1) in a form of a switched control system:

$$
\begin{aligned}
& x(k+1)=A_{s(k)} x(k)+B_{s(k)} u(k)+C_{s(k)}(k), \\
& s(k)=f(x(k), u(k)) .
\end{aligned}
$$

Here the state vector $x \in \mathcal{X}$ and the input vector $u \in \mathcal{U}$ stand for vehicle densities and on-ramp demands, respectively:

$$
x=\left(\rho_{0}, \rho_{1}, \ldots, \rho_{n-1}\right), u=\left(u_{0}, u_{1}, \ldots, u_{n-1}\right) .
$$


The sets $\mathcal{X} \in \mathcal{R}^{n}$ and $\mathcal{U} \in \mathcal{R}^{n}$ respect minimum and maximum values for densities and on-ramp demands. The switching variable $s \in \mathcal{S}=\{1,2, \ldots, m\}$ indicates a mode of the system, and it determines terms in the matrices $A^{n \times n}$, $B^{n \times n}$ and for the vector $C^{n \times 1}(k)$. The vector $C^{n \times 1}(k)$ consist of the boundary functions $\bar{D}(k)$ and $\bar{S}(k)$. Notice, that $m$ is a total number of all possible modes generated by nonlinear formulas for mainstream and on-ramp flows.

\section{PROBLEM SETTING}

In this section, we pose an optimization problem to find balanced equilibrium points for the system described by (7). Before we set up the problem, we introduce a few definitions.

\section{A. Balanced Equilibrium Points for CTM}

The feasible set for the optimization problem will be limited to a set of the equilibrium points for CTM. We define this set below.

Definition: The set of equilibrium points for CTM, represented by (7), is a set of pairs $(x, u)$ that under the fixed boundary conditions they solve the following equations:

$$
\begin{aligned}
& \left(A_{s}-\mathbf{I}\right) x+B_{s} u+C_{s}=0, \\
& s=f(x, u) .
\end{aligned}
$$

Here I stands for the identity matrix. In terms of (1) the steady state is reached when for every cell, the total flow entering and leaving are equal. Notice, that vectors $C_{s}$ becomes constant, when boundary conditions have fixed value, i.e.: $\bar{D}(k)=\bar{D}$ and $\bar{S}(k)=\bar{S}$. Components of the equilibrium point $x$ and $u$ will be called later as equilibrium density and equilibrium input flow, respectively.

Two types of density distributions are of our special interest. We will call these distributions as exactly balanced and near balanced (see Fig. 3). We introduce a set of admissible balanced densities:

$$
\mathcal{C}=\left\{c: 0 \leq c \leq \min \left\{\bar{\rho}_{i}\right\}_{i=0}^{n-1}\right\}
$$

Definition: An exactly balanced density $x^{e b}$ is a vector that for some $c \in \mathcal{C}$ the following condition is fulfilled:

$$
x_{i}^{e b}=c, \forall i=0,1, \ldots, n-1 .
$$

By exactly balanced equilibrium point $x^{e b_{-} e q}$ we will call the equilibrium density that is exactly balanced. In [13], it was shown, how the structure of equilibrium sets corresponds to the cell parameters. It was proven that if some condition on these parameters does not hold, then the set of exactly balanced equilibrium points is empty. Therefore, for the general case, instead of exactly balanced equilibrium, we should rather consider an equilibrium which is near balanced. Roughly speaking, by this near balanced equilibrium density, we will mean a density distribution that tends to be exactly balanced, but on the other hand, it stays in the equilibrium set. Formally we can define it as follows:

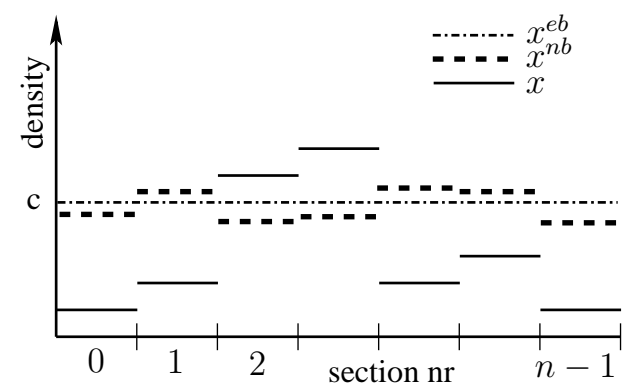

Fig. 3. Exemplary distributions of density in freeway cells. Solid line, dashed line and dash-dotted line corresponds to unbalanced, near balanced and exact balanced case, respectively.

Definition: A near balanced equilibrium density $x^{n b_{-} e q}$ is a vector that for some $c \in \mathcal{C}$ and some norm $\|\cdot\|$ solves the following optimization problem:

$$
x^{n b_{-} e q}=\operatorname{argmin}_{x \in \mathcal{X}}\|x-c \mathbf{1}\| .
$$

under the constraints (9). Here 1 stands for all-ones vector.

\section{B. Constraints Representation}

A set of equilibrium points, as defined in (9), represents an irregular shape composed of hyperplanes. This kind of constraints is extremely difficult to be treated globally in optimization problems. The idea will be to decompose the global optimization problem into a set of local sub-problems. For that purpose, we now decompose the equilibrium set.

For every $s \in \mathcal{S}$ we define the set:

$$
\Delta_{s}=\{(x, u): s=f(x, u)\} .
$$

The function $s=f(x, u)$ results from $\operatorname{mid}\{\cdot\}$ and $\min \{\cdot\}$ terms in (3-5). Therefore, by introducing the relevant inequalities, that describe $\Delta_{s}$, i.e. by rewriting $\Delta_{s}$ as:

$$
\Delta_{s}=\left\{(x, u): K_{s} x+L_{s} u+M_{s} \leq 0\right\} .
$$

we can reformulate (9) as follows:

$$
\begin{aligned}
\left(A_{s}-\mathbf{I}\right) x+B_{s} u+C_{s} & =0, \\
K_{s} x+L_{s} u+M_{s} & \leq 0, \quad s \in \mathcal{S} .
\end{aligned}
$$

Here the matrices $K_{s}, L_{s}$ and the vector $M_{s}$ are constant and of size resulting from a design of $\Delta_{s}$. To illustrate how to build (15) we give an example for the case of two-cell system. Using (1)-(6) we can write:

$$
\begin{aligned}
\text { for } s=1: & \bar{D}+u_{0}=\frac{1}{\bar{\beta}_{0}} D_{0}, D_{0}+u_{1}=\frac{1}{\bar{\beta}_{1}} D_{1}, \\
& \bar{D}+u_{0} \leq S_{0}, D_{0}+u_{1} \leq S_{1}, D_{1} \leq \bar{S}, \\
\text { for } s=2: & S_{0}=\frac{1}{\bar{\beta}_{0}} D_{0}, D_{0}+u_{1}=\frac{1}{\bar{\beta}_{1}} D_{1}, \\
& \bar{D}+u_{0} \geq S_{0}, D_{0}+u_{1} \leq S_{1}, D_{1} \leq \bar{S},
\end{aligned}
$$

and so forth . 
For the free flow case the insertion of the formulas for Demand and Supply functions for $s=1$ results as follows:

$$
\begin{array}{r}
{\left[\begin{array}{cc}
-v_{0} & 0 \\
\bar{\beta}_{0} v_{0} & -v_{1}
\end{array}\right]\left[\begin{array}{l}
x_{0} \\
x_{1}
\end{array}\right]+\left[\begin{array}{ll}
1 & 0 \\
0 & 1
\end{array}\right]\left[\begin{array}{l}
u_{0} \\
u_{1}
\end{array}\right]+\left[\begin{array}{c}
\bar{D} \\
0
\end{array}\right]=0,} \\
{\left[\begin{array}{cc}
w_{0} & 0 \\
\bar{\beta}_{0} v_{0} & w_{1} \\
0 & \bar{\beta}_{1} v_{1} \\
v_{0} & 0 \\
0 & v_{1}
\end{array}\right]\left[\begin{array}{l}
x_{0} \\
x_{1}
\end{array}\right]+\left[\begin{array}{ll}
1 & 0 \\
0 & 1 \\
0 & 0 \\
0 & 0 \\
0 & 0
\end{array}\right]\left[\begin{array}{l}
u_{0} \\
u_{1}
\end{array}\right]+\left[\begin{array}{c}
\bar{D}-w_{0} \bar{\rho}_{0} \\
-w_{1} \bar{\rho}_{1} \\
-\bar{S} \\
-F_{0} \\
-F_{1}
\end{array}\right] \leq 0 .}
\end{array}
$$

\section{Optimization Problems}

As we stated in the introduction, the goal is to find optimal balanced equilibrium points. So far, we have defined balanced equilibria. We have also mentioned that for the general case, these equilibria are near balanced instead of exactly balanced. Now, we want to put additional requirements. We want to design near balanced density that maximizes both the Total Travel Distance and total input flows. We will call this state as optimally balanced. The optimization will be executed in two steps. At the first step, we will find the exactly balanced density, that maximizes TTD. According to the previous definitions, we search for the optimal value of $c$ so that TTD is maximized. We denote this optimal value as $c^{*}$. The second step will be to determine the optimal equilibrium density $x^{*}$ and the optimal equilibrium input flow $u^{*}$ such that $x^{*}$ tends to the value $c^{*}$, and $u^{*}$ is maximized. Note that at both steps we will solve static optimization problems since we consider the steady states only.

Now we will pose the first optimization problem to find $c^{*}$. Under the assumption that the system is in the steady state, for the total length of a freeway $\sum_{i=0}^{n-1} l_{i}$ and for some time interval $T$, the Total Travel Distance is as follows:

$$
\operatorname{TTD}=\int_{0}^{\sum_{i=0}^{n-1} l_{i}} \int_{0}^{T} \Phi(\kappa, t) \mathrm{d} \kappa \mathrm{d} t=T \sum_{i=0}^{n-1} \bar{\Phi}_{i} l_{i},
$$

where $\Phi(\kappa, t)$ and $\bar{\Phi}_{i}$ stand for the total flow in spacetime domain and the mean flow for the cell $i$, respectively. According to the Fundamental Diagram (Fig. 2) we can rewrite (18) in the following form:

$$
\operatorname{TTD}=T \sum_{i=0}^{n-1} \min \left\{v_{i} \rho_{i}, w_{i}\left(\bar{\rho}_{i}-\rho_{i}\right)\right\} l_{i} .
$$

Assuming, that the density is exactly balanced, we can consider the following problem:

\section{Problem 1:}

$$
\begin{aligned}
\text { Find } c^{*} & =\operatorname{argmax}_{c \in \mathcal{C}} J_{1}(c), \\
J_{1}(c) & =\sum_{i=0}^{n-1} \min \left\{v_{i} c, w_{i}\left(\bar{\rho}_{i}-c\right)\right\} l_{i} .
\end{aligned}
$$

The method for solving this problem will be presented in the next section.
When $c^{*}$ is determined, we can formulate the second optimization problem to find the optimal balanced equilibrium point. To be consistent with (7) and (9) we denote the optimal equilibrium density and optimal equilibrium input flow as $x^{*}$ and $u^{*}$, respectively. We pose the following problem:

\section{Problem 2:}

$$
\begin{gathered}
\text { Find } \quad\left(x^{*}, u^{*}\right)=\operatorname{argmin}_{x \in \mathcal{X}, u \in \mathcal{U}} J_{2}(x, u), \\
J_{2}(x, u)=\left(x-c^{*} \mathbf{1}\right)^{T} Q\left(x-c^{*} \mathbf{1}\right)-u^{T} R u \\
\text { under } \quad\left(A_{s}-\mathbf{I}\right) x+B_{s} u+C_{s}=0, \\
K_{s} x+L_{s} u+M_{s} \leq 0, s \in \mathcal{S} .
\end{gathered}
$$

The objective function $J_{2}$ is composed of two quadratic terms. The first one is to provide near balanced density while the second one is to maximize and weight the input flows. Matrices $Q^{n \times n}$ and $R^{n \times n}$ are assumed to be positivedefinied symmetrical. Detailed study on the convexity of $J_{2}$ will be provided in the section $\mathrm{V}$.

\section{SOLUTION OF THE PROBLEM 1}

In this section, we present a method for determining the desired density $c^{*}$. A procedure is based on decomposition of nonlinear Problem 1 into a set of simple linear problems.

Let us consider the expression in (20):

$$
\sum_{i=0}^{n-1} \min \left\{v_{i} c, w_{i}\left(\bar{\rho}_{i}-c\right)\right\} l_{i}
$$

It can return $2^{n}$ different linear functions of $c$. Let $j=$ $1,2, \ldots, 2^{n}$ be the index for a sequence of all possible modes of (22). For every mode, we can introduce constraints:

$$
\begin{gathered}
\eta_{j} c+\gamma_{j} \leq 0 \\
\eta_{j}=\left(\eta_{j, 0}, \eta_{j, 1}, \ldots, \eta_{j, n-1}\right), \gamma_{j}=\left(\gamma_{j, 0}, \gamma_{j, 1}, \ldots, \gamma_{j, n-1}\right)
\end{gathered}
$$

that describe feasible set of $c$. Notice that the sign of $\eta_{j, i}$ is determined as follows:

$$
\begin{aligned}
& \eta_{j, i}<0 \text { in the case of } v_{i} c \geq w_{i}\left(\bar{\rho}_{i}-c\right) \\
& \eta_{j, i}>0 \text { in the case of } v_{i} c \leq w_{i}\left(\bar{\rho}_{i}-c\right)
\end{aligned}
$$

This fact will be used in the computational algorithm (see the Appendix). Now, instead of the Problem 1 we can consider a sequence of linear optimization problems:

$$
\begin{aligned}
& \text { Find } \quad c_{j}^{*}=\operatorname{argmax}_{c \in \mathcal{C}} c \Theta_{j}+\Psi_{j} \\
& \text { under } \eta_{j} c+\gamma_{j} \leq 0, \quad j=1,2, \ldots 2^{n} .
\end{aligned}
$$

Here $\Theta_{j}$ and $\Psi_{j}$ stand for relevant terms of (22). After solving (25) we can extract the optimal solution for the original Problem 1:

$$
c^{*}=c_{z}^{*},
$$

where $z$ is such that:

$$
J_{1}\left(c_{z}^{*}\right)=\max \left\{J_{1}\left(c_{j}^{*}\right)\right\}_{j=1}^{2^{n}} .
$$


Note that (25) can be simplified as follows:

$$
\begin{aligned}
& \text { if } \Theta_{j}>0 \quad c_{j}^{*}=\operatorname{argmax}_{c \in \mathcal{C}} c, \\
& \text { if } \Theta_{j}<0 \quad c_{j}^{*}=\operatorname{argmin}_{c \in \mathcal{C}} c, \\
& \text { if } \Theta_{j}=0 \quad c_{j}^{*}=c \\
& \eta_{j} c+\gamma_{j} \leq 0, \quad j=1,2, \ldots, 2^{n} .
\end{aligned}
$$

Remark: If $\Theta_{j}=0$ then there is no unique optimal solution for (25). In that case, we can choose any value, that respects constraints.

The problem (28) can be solved by standard linear programming methods. However, using some facts on the structure of the constraints, we have developed an easy for implementation computational algorithm. It is presented in the Appendix.

\section{SOLUTION OF THE PROBLEM 2}

In this section, we focus on the Problem 2. The global optimization problem will be treated as the set of local convex quadratic problems that will be solved by using the Lagrange Dual Functions.

\section{A. Reformulation of the Problem 2}

Using the equilibrium equation (9) we can reformulate the Problem 2, so it can be reduced to finding $u^{*}$. Moreover, the problem can be decomposed according to the constraints decomposition introduced previously.

In order to guarantee the existence of solution of the optimization problem, we consider only these modes $s$, for which $x$ in the equilibrium equation:

$$
\left(A_{s}-\mathbf{I}\right) x+B_{s} u+C_{s}=0
$$

can be expressed as a function of $u$. Thus, we limit our interest to the modes $s \in \mathcal{S}^{*}$, where:

$$
\mathcal{S}^{*}=\left\{s \in \mathcal{S}:\left(A_{s}-\mathbf{I}\right)^{-1} \text { exist and } B_{s} \text { is non-zero }\right\} .
$$

Then we can rewrite (29) as follows:

$$
x=\left(\mathbf{I}-A_{s}\right)^{-1}\left(B_{s} u+C_{s}\right) .
$$

By introducing:

$$
W_{s}=\left(\mathbf{I}-A_{s}\right)^{-1} B_{s}, V_{s}=\left(\mathbf{I}-A_{s}\right)^{-1} C_{s} .
$$

the Problem 2 can be decomposed into the following subproblems:

$$
\begin{aligned}
& \text { Find } \quad u_{s}^{*}=\operatorname{argmin}_{u \in \mathcal{U}} \bar{J}_{2}(u), \\
& \qquad \bar{J}_{2}(u)=\left(W_{s} u+V_{s}-c^{*} \mathbf{1}\right)^{T} Q\left(W_{s} u+V_{s}-c^{*} \mathbf{1}\right)+ \\
& \quad-u^{T} R u \\
& \text { under } K_{s}\left(W_{s} u+V_{s}\right)+L_{s} u+M_{s} \leq 0, \quad s \in \mathcal{S}^{*} .
\end{aligned}
$$

The optimal solution for the original Problem 2 is given as:

$$
\left(x^{*}, u^{*}\right)=\left(W_{z} u_{z}^{*}+V_{z}, u_{z}^{*}\right),
$$

where $z$ fulfils the following equation:

$$
\bar{J}_{2}\left(u_{z}^{*}\right)=\min \left\{\bar{J}_{2}\left(u_{s}^{*}\right)\right\}_{s \in \mathcal{S}^{*}} .
$$

\section{B. The Lagrange Dual Problem}

Here we present a brief summary on the basics of the Lagrange Dual Problem (for more details see [18]). By $p^{*}$ we denote the optimal value for some objective $J(u)$ under the constraints $g(u) \leq 0$. Let us introduce the Lagrangian:

$$
\mathcal{L}(u, \lambda)=J(u)+\lambda^{T} g(u)
$$

and the Lagrange Dual Function:

$$
q(\lambda)=\min _{u \in \mathcal{U}} \mathcal{L}(u, \lambda) .
$$

The Lagrange Dual Problem is the maximization of the Lagrange Dual Function:

$$
q^{*}=\max _{\lambda \succeq 0} q(\lambda) .
$$

If the problem is convex and Slater's condition is fulfilled, then the equality holds

$$
q^{*}=p^{*} .
$$

Slater's condition is a constraints qualification, and it holds for affine $g(u)$. Therefore, instead of the original problem, we can solve the Dual Problem.

To solve the Dual Problem, we can use the method of the steepest descent with the following updates:

$$
\begin{aligned}
& u^{+}=u-\alpha_{1} \nabla_{u} \mathcal{L}, \text { and } u \in \mathcal{U}, \\
& \lambda^{+}=\lambda+\alpha_{2} \nabla_{\lambda} \mathcal{L}, \text { and } \lambda \succeq 0 .
\end{aligned}
$$

Here $\alpha_{1}>0$ and $\alpha_{2}>0$. The convergence is assured by the convexity.

C. The convexity condition and an application of the Lagrange Dual Functions for the Problem 2

Due to the affine constraints in (33) the Slater's condition is fulfilled. To apply the Dual Problem there remains the question of convexity of $\bar{J}_{2}(u)$.

Explicit formula for $\bar{J}_{2}(u)$ is given below:

$$
\begin{aligned}
\bar{J}_{2}(u)= & u^{T}\left(W_{s}^{T} Q W_{s}-R\right) u+ \\
& 2\left(V_{s}^{T} Q W_{s}-c \mathbf{1}^{T} Q W_{s}\right) u+ \\
& -2 c V_{s}^{T} Q \mathbf{1}+V_{s}^{T} Q V_{s}+c^{2} \mathbf{1}^{T} Q \mathbf{1} .
\end{aligned}
$$

We can easily observe that if the matrices $W_{s}$ and $V_{s}$ are continuous with respect to $u$, then $\bar{J}_{2}(u)$ will be continuously differentiable function and the problem can be classified as an optimization of quadratic spline. Similar problems were previously studied, for example, in [17]. Here we recall one fundamental fact. The piecewise convexity does not imply the global convexity. Therefore, the decomposition into local problems seems to be reasonable not only because of complex constraints, but also for the convexity. In fact, the relation in (31) between $x$ and $u$ may not be continuous in the points where $s \in S^{*}$ switches. As a consequence $\bar{J}_{2}(u)$ is only piece-wise continuous. This is another motivation for the local treatment of the Problem 2.

From (41) we immediately conclude that piecewise convexity holds iff for every $s$ the inequality

$$
W_{s}^{T} Q W_{s}-R \succeq 0
$$


is fulfilled. Note that this condition imposes a requirement on the choice of matrices $Q$ and $R$ and therefore, it limits a trade-off between balancing and input flows maximization.

Respecting the convexity condition (42) we can now solve the sub-problems (33) by using the Dual Functions and the updates (40). Below, we give the formulas for the Lagrangian and its gradients:

$$
\begin{aligned}
\mathcal{L}(u, \lambda)= & \bar{J}_{2}(u)+\lambda^{T}\left(K_{s}\left(W_{s} u+V_{s}\right)+L_{s} u+M_{s}\right) \\
\nabla_{u} \mathcal{L}= & 2\left(W_{s}^{T} Q W_{s}-R\right) u+2 W_{s}^{T} Q\left(V_{s}-c \mathbf{1}\right)+ \\
& +\left(W_{s}^{T} K_{s}^{T}+L_{s}^{T}\right) \lambda \\
\nabla_{\lambda} \mathcal{L}= & K_{s}\left(W_{s} u+V_{s}\right)+L_{s} u+M_{s} .
\end{aligned}
$$

\section{CONCLUDING REMARKS}

In this paper, a method for finding optimal balanced equilibrium points for the Cell Transmission Traffic Model has been presented. A two-step procedure has enabled us to solve the problem by using efficient computational methods. The first nonlinear problem has been simplified to set of linear sub-problems. Using the facts on the structures of the constraints, an easy for implementation computational algorithm has been proposed. The second problem has required decomposition due to the complex constraints and the discontinuity of the objective function. This problem has been treated as a set of convex sub-problems, that can be solved by using the Dual Functions. A convex formulation gives us an opportunity to reformulate the method so to be solved in a distributed manner. The application of the proposed algorithms for Grenoble South Ring will be addressed in future works.

\section{ACKNOWLEDGMENTS}

The research leading to these results has received funding from the European Union Seventh Framework Programme [FP7/2007-2013] under grant agreement $n^{0} 257462$ HYCON2 Network of excellence.

\section{REFERENCES}

[1] A. Hegyi, B. De Schutter, J. Hellendroon, T. van den Boom, Optimal Coordination of Ramp Metering and Variable Speed Control - An MPC Approach, IEEE American Control Conference, 2002, pp 36003605.

[2] X. Y. Lu, T. Z. Qiu, P. Varaiya, R. Horowitz, S. E. Shladover, Combining Variable Speed Limits with Ramp Metering for Freeway Traffic Control, IEEE American Control Conference, 2010, pp 22662271.

[3] G. Gomes, R. Horowitz, Optimal Freeway Ramp Metering Using the Asymmetric Cell Transmission Model, Transportation Research Part $C$, vol. 14, 2006, pp 244-262.

[4] D. Jacquet, C. Canudas-de-Wit, D. Koenig, Optimal Control of Systems of Conservation Laws and Application to Non-Equilibrium Traffic Control, Proceedings of the 13th IFAC Workshop on Control Applications of Optimisation, 2006.

[5] A. Hegyi, B. De Schutter, J. Hellendroon, Model Predictive Control for Optimal Coordination of Ramp Metering and Variable Speed Limits, Transportation Research Part C, vol. 13, 2005, pp 185-209.

[6] A. Di Febbraro, T. Parisini, S. Sacone, R. Zoppoli, Neural Approximation for Feedback Optimal Control of Freeway Systems, IEEE Transactions on Vehicular Technology, vol. 50(1), 2001, pp 302-312.
[7] A. Alessandri, A. Di Febbraro, A. Ferrara, E. Punta. Optimal Control of Freeways via Speed Signalling and Ramp Metering, Control Engineering Practice, vol. 6, 1998, pp 771-780.

[8] M. Papageorgiou, H. Hadj-Salem, J. M. Blosseville, ALINEA: A Local Feedback Control Law for On-ramp Metering, Transportation Research Record, vol. 1320, 1991, pp 58-64.

[9] D. P. Masher, D. W. Ross, P. J. Wong, P. L. Tuan, P. L. Ziedler, S. Peracek, Guidelines for Design and Operation of Ramp Control Systems, SRI, Menid Park, CA, Stanford Res. Inst. Rep. NCHRP $3-$ 22, SRI Project 3340, 1975.

[10] Y. H. Chiang, J. Ch. Yuang, Decentralized Control of Freeway Traffic Flow with Neighboring Cellular Information, IEEE International Conference on Systems, Man and Cybernetics, 2006, pp 2547-2552.

[11] M. Baric, F. Borelli, Distributed Averaging with Flow Constraints, IEEE American Control Conference, 2011, pp 4834-4839.

[12] G. Gomes, R. Horowitz, A. A. Kurzhanskiy, P. Varaiya, J. Kwon, Behavior of The Cell Transmission Model and Effectiveness of Ramp Metering, Transportation Research Part C, vol. 16, 2008, pp 485-513.

[13] D. Pisarski and C. Canudas-de-Wit, Analysis and Design of Equilibrium Points for the Cell-Transmission Traffic Model, to appear in IEEE American Control Conference, 2012.

[14] Grenoble Traffic Lab, http://necs.inrialpes.fr/pages/reseach/gtl.php

[15] C. Daganzo, The Cell Transmission Model: A Dynamic Representation of Highway Traffic Consistent with the Hydrodynamic Theory, Transportation Research Part B, vol. 28, 1994, pp 269-287.

[16] R. J. LeVeque, Numerical Methods for Conservation Laws, Birkhauser Verlag, Basel, 1992.

[17] F. V. Louveaux, Piecewise Convex Programs, Mathematical Programming, vol. 15, 1978, pp 53-62.

[18] S. Boyd, L. Vandenberghe, Convex Optimization, Cambridge University Press, New York, 2004.

\section{APPENDIX}

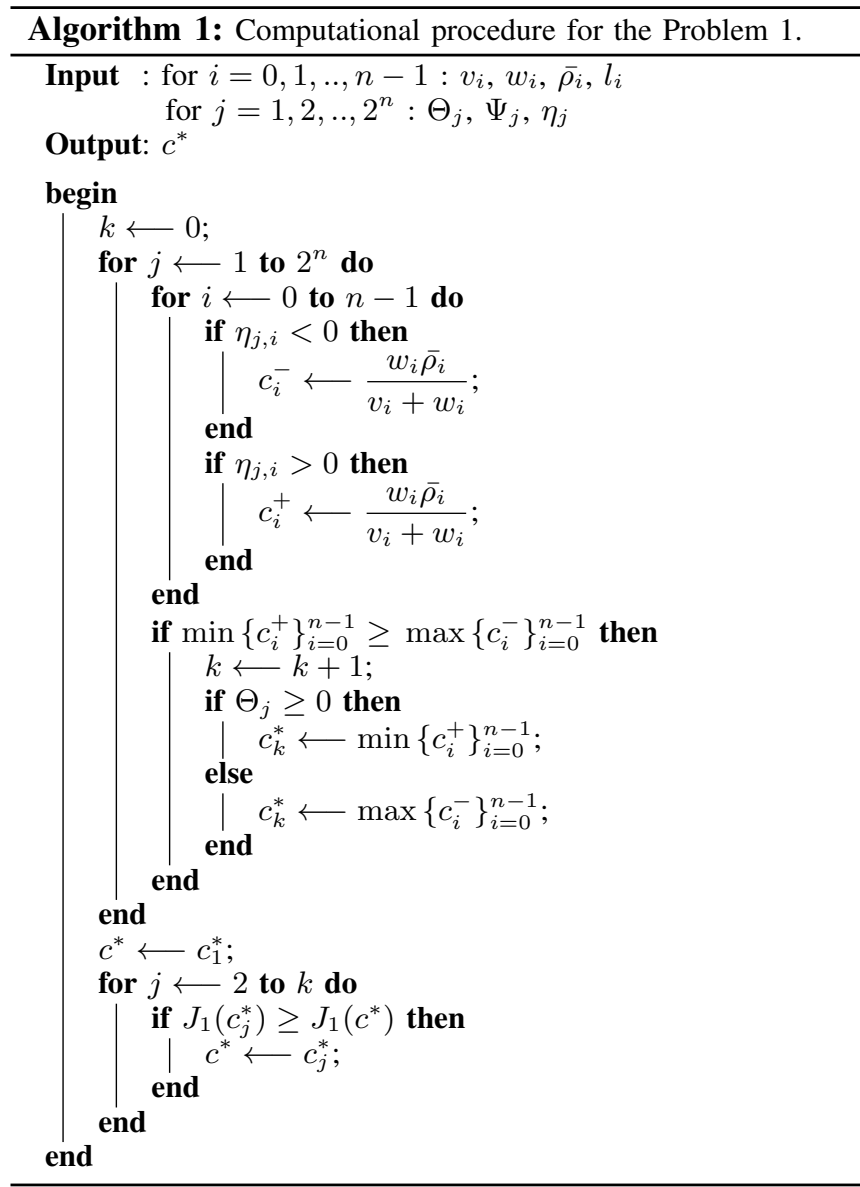

